\title{
A Preliminary Study on the Effect of Low Temperature Kinetics on Engine Modeling
}

\author{
Arturo de Risi, Domenico Laforgia \\ Università di Lecce - Dipartimento di Ingegneria dell'Innovazione,73100 via Arnesano, Lecce - Italy \\ Teresa Donateo \\ ISUFI - Innovative Materials and Technologies - Università di Lecce,73100 via Monteroni, Lecce - Italy
}

\section{ABSTRACT}

Modeling autoignition in diesel engines is a challenging task because of the wide range of equivalence ratios over which it takes place. A variety of detailed autoignition models has been proposed in literature for different fuels. Since these models include about one thousand chemical reactions and more than one hundred species, their application to CFD engines simulations requires a very high computational time, so that they are of no practical interest. In order to lower the computational time, a number of reduced models has been developed including the shell model, which is one of the most used. This model does not take into account low temperature kinetics and consists of seven reactions and three radicals. The use of this model in engine simulations shows its limits when applied to delayed injections because of the predominant influence of the low temperature kinetics. A modified version of the shell model is proposed in the present study. It includes the effect of low temperature kinetics by the addition of two more radicals and three new kinetics reactions. The model has been implemented in a modified version of the KIVA3V code. The performance of the new kinetic scheme has been investigated by computing the ignition delay at different operating conditions in bomb like simulations. The investigated operating conditions were obtained by changing gas temperature and pressure, air to fuel ratio (i.e., oxygen concentration). The effect of the pre-exponential factor in the rate of production of the intermediate agent has also been investigated. The model has also been applied to predict autoignition of a commercial small bore direct injection diesel engine for different injection timings. Results showed that, for delayed injections, the new model was able to better predict the heat released and the pressure traces. The influence of the modified shell model on engine emissions has also been analyzed.

\section{INTRODUCTION}

In compression ignition engines, the combustion process starts from the autoignition of the fuel-oxygen mixture and the ignition delay has a strong influence on engine performance and pollutant emissions. In fact, the two main parameters characterizing the autoignition process are the initial temperature at which autoignition can develop and the time delay before ignition. The detailed kinetic mechanism of the autoignition process includes about one thousand chemical reactions and over a hundred species. Thus, its application to CFD simulations codes requires high computational times.

A group of researchers from Shell Research, Ldt. developed a reduced kinetic scheme introducing generic species with kinetic rate constants deduced from experimental data [1] in order to predict knock in spark ignition engines. This model was modified to assure mass conservation [3] and to adjust kinetic rates to fit experimental data [2]. Theobald [4] applied the shell model to diesel engine autoignition phenomena by modifying the kinetic rate. Since then, the shell model has been widely applied in CFD simulations of diesel engine [5] and has been shown to successfully predict ignition processes happening before top dead center.On the other hand, this reduced scheme fails to predict both the ignition delay and the pressure rate of change in the case of delayed injections. This reveals that the shell model does not adequately describe the ignition phenomena which take place at low temperatures.

To improve the prediction capability of the model, Cox et al. [6] and Hu et al [7] increased the number of reactions introducing the chemistry controlling the hydrocarbon oxidation process at low temperatures. But the main advantage of the shell model is the low number of species and reactions, which makes this model suitable for CFD applications. Thus, improvements should not substantially increase the complexity of the model. To achieve this twofold goal (i.e., a simple scheme able to predict low temperature hydrocarbon ignition) Gaballo [8] 
developed a modified version of the shell model by adding two more species and three kinetic reactions.

In the present investigation, this modified version of shell model has been implemented in the KIVA3V code [11] and its capability to predict the low temperature autoignition process has been checked. Experimental data from a small bore direct injection diesel engine have been used for comparison. Moreover, the numerical pressure traces obtained with the modified version of the shell model have been compared with the results of the original shell model and the effect of the low temperature chemistry on the soot and NOx engine emissions has been also investigated.

\section{THE KIVA 3V CODE}

Numerical simulations have been performed by using a modified version of the KIVA $3 \mathrm{~V}$ code whose improved models for spray, turbulence, combustion process and emissions mechanisms of formation are shortly described in the present section. A complete description of the models can be found in [9].

\section{TURBULENCE MODEL}

The modified RNG $k-\varepsilon$ model proposed by Han et al. [10] has been used to simulate the in-cylinder turbulent flow field. This model is able to predict the large scale structures produced by the squish flows and generated by the spray. It is characterized by the presence of an extra term in the dissipation equation that accounts for the flow compressibility.

\section{SPRAY MODELS}

The fuel injection process is modeled by using the blob injection model: the liquid fuel is represented by big drops, or "blobs", having a radius equal to the effective nozzle radius [12]. A breakup model based on the KelvinHelmholtz $(\mathrm{H}-\mathrm{K})$ and Reyleigh-Taylor $(\mathrm{R}-\mathrm{H})$ instability criteria has been adopted to describe the drop break-up process after injection [13]. According to this model, breakup is due to $\mathrm{K}-\mathrm{H}$ instability until the spray reaches the breakup length, then breakup occurs due to both $\mathrm{K}-\mathrm{H}$ and R-T mechanisms.

\section{COMBUSTION MODELS}

The combustion chemistry is modeled with the laminar and turbulent characteristic time combustion model proposed by Abraham et al. [14]. For engine applications, the combustion model is activated when a threshold temperature of $1100 \mathrm{~K}$ is reached or a determined amount of combustion products is generated by the ignition process.

\section{EMISSIONS MODELS}

The soot emission model adopted in the present study is the Hiroyasu formation model [15] combined with the Nagle and Strickland-Constable oxidation model [16]. NO emissions are modeled with the Zel'dovich mechanism.

\section{THE SHELL MODEL}

The shell model is a reduced kinetic scheme which accounts for the large number of radicals and intermediate species involved in hydrocarbon oxidation by introducing generic molecular species. In particular, species which play a similar role in the ignition chemistry are considered as a single entity. For instance, individual radicals are not specifically identified and the mechanism is expressed in terms of the total radicals concentration [R]. The kinetic scheme of the shell model consists of eight reactions given by equations (1) - (8). In these reactions $R H$ represents hydrocarbon fuel $\left(C_{n} H_{2 m}\right)$, $\mathrm{R}$ are the radical species, $\mathrm{B}$ the branching agent, $\mathrm{Q}$ the intermediate agent, $\mathrm{P}$ the products consisting of $\mathrm{CO}, \mathrm{CO}_{2}$ and $\mathrm{H}_{2} \mathrm{O}$.

\begin{tabular}{|llll|}
\hline Description & Reaction & $\begin{array}{l}\text { Rate } \\
\text { coefficient }\end{array}$ & \\
Initiation & $R H+\mathrm{O}_{2} \longrightarrow 2 R$ & $k_{q}$ & $(1)$ \\
Propagation & $R \longrightarrow R+P$ & $k_{p}$ & $(2)$ \\
Propagation & $R \longrightarrow R+B$ & $f_{1} k_{p}$ & $(3)$ \\
Propagation & $R+Q \longrightarrow R+B$ & $f_{2} k_{p}$ & $(4)$ \\
Propagation & $R \longrightarrow R+Q$ & $f_{4} k_{p}$ & $(5)$ \\
Branching & $B \longrightarrow 2 R$ & $k_{b}$ & $(6)$ \\
Termination & $R \longrightarrow$ out & $f_{3} k_{p}$ & $(7)$ \\
Termination & $2 R \longrightarrow$ out & $k_{t}$ & $(8)$ \\
\hline
\end{tabular}

Table 1 - The shell kinetic scheme

The rate coefficients $\mathrm{kq}, \mathrm{kt}$ and $\mathrm{kb}$ take the Arrhenius form of eq.(9).

$$
k_{j}=A_{j} \exp \left(-E_{j} / R T\right)
$$

$\mathrm{kp}$ expresses the chain propagation rate as a function of the total radical concentration [R] and it is compounded from three separate rates:

$$
k_{p}=\frac{1}{\frac{1}{k_{p 1}}\left[O_{2}\right]+\frac{1}{k_{p 2}}+\frac{1}{k_{p 3}}[R H]}
$$

where $k p_{1}, k p_{2}, k p_{3}$ are the rate coefficients for the propagation steps which do not directly appear in the kinetic scheme of the shell model. 
Halstead et al. [2] suggested that the reaction parameters fi can be written as:

$$
f_{i}=A_{f i} \exp \left(-E_{f i} / R T\right)\left[O_{2}\right]^{x i}[R H]^{y i}, i=1,4
$$

where xi and yi are empirical parameters to be tuned to fit experimental data. The non-reactive species in the termination equations are assumed to be nitrogen.

Because of the use of generic species, the equations of the shell kinetic scheme don't conserve mass. Thus, the model cannot be used in the original formulation for CFD simulations. Schäpertöns and Lee [3] suggested that equations (2), (3) and (5) should be combined in a single propagation step to assure mass conservation. This results in the following mass balanced reaction:

$$
\begin{aligned}
& R+(\lambda+1)\left(R H / m+p O_{2}\right) \rightarrow q P+ \\
& f_{1} B+f_{4} Q+R
\end{aligned}
$$

where $\lambda=\left(f_{1} M_{B}+f_{4} M_{Q}\right) /\left(M_{R H} / m+p M_{O_{2}}\right) \quad$ is the stoichiometric coefficient, $p=[n(2-\gamma)+m] / 2 m$, $q=(n / m+1)$ and $\gamma=0.67$ is the relative yield of $\mathrm{CO}$ and $\mathrm{CO}_{2}$.

The shell model requires 26 rate and concentration parameters to be determined for each fuel. By comparison with experimental data, Halstead et al. [2] derived the kinetic parameters for fuel with different Octane Number (RON).

Since the basic chemistry governing autoignition is indipendent of the specific hydrocarbon fuel, Theobald [4] applied the shell model to diesel autoignition processes without changing the structure of the model. However, the kinetic parameters have to be changed to fit the experimental data of heavier hydrocarbons which show a shorter ignition delay, compared with gasoline fuels. Thus, the kinetic parameters found by Halstead et al. [2] have been modified by Theobald et al. [4] to switch from lighter hydrocarbons typically found in gasoline to higher hydrocarbons more similar to diesel fuels. Hardenberg et al. [17] showed that the activation energy of the ignition process depends on the fuel cetane number. Thus, Ayoub et al. [18] suggested that the reaction parameter Ef4 should be corrected by using eq. (13).

$E_{f 4}^{\prime}=E_{f 4} \frac{65}{C N+25}$

\section{IMPROVEMENTS TO THE SHELL MODEL}

Even if it is widely used for diesel engine CFD simulations, the shell model fails to predict the ignition delay in the case of delayed injections which correspond to combustion starting more than five-ten degrees after top dead center. This is because the shell model does not take into account the low temperature hydrocarbon oxidation which has been shown by several authors $[6,7]$ to play an important role at relatively low temperatures. The low temperature alkane oxidation scheme can be written [8] as:

$$
\begin{aligned}
& \text { cyclic ether }+\mathrm{OH} \\
& \text { or } \beta \text { scission }+\mathrm{HO}_{2} \\
& \uparrow
\end{aligned}
$$

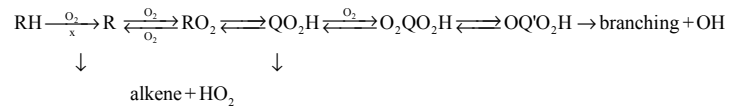

From kinetics considerations Gaballo [8] selected three reactions from scheme (14) which are believed to be the most influent on the hydrocarbon low temperature ignition process. These reactions involve two more species than the shell model: the hydroperoxyalkyl $\mathrm{QOOH}$ and the peroxyalkyl hydroperoxy radical $\mathrm{O}_{2} \mathrm{QOOH}$. Based on the results of Gaballo, the authors included the low temperature kinetics in the shell model by adding Eqs. (15)-(17). In these equations, $Q_{1}$ and $B_{1}$ are generic species which can be referred to $\mathrm{QOOH}$ and $\mathrm{O}_{2} \mathrm{QOOH}$, respectively. In order to assure mass conservation, eq. (15) has been combined with eq. (2), (3) and (5), using the Schäpertöns and Lee [3] manner. The molecular weight of the generic species involved in the complete scheme is calculated to accomplish mass balancing.

\begin{tabular}{lll}
\hline Description & Reaction & Rate coefficient \\
Propagation & $R \rightarrow Q_{1}$ & $k_{a}$ \\
Propagation & $Q_{1}+O_{2} \rightarrow B_{1}$ & $k_{q 1}$ \\
Branching & $B_{1} \rightarrow 2 R$ & $k_{b_{1}}$
\end{tabular}

Table 2 - The kinetic scheme of the modified shell model

The model developed by the authors, henceforth referred as the modified shell model, is not expected to increase the computational time of CFD engine simulations thanks to the reduced number of reactions and chemical species added to the original shell model. Tests on engine simulations have shown an increase of the computational time by about $1 \%$.

\section{DETERMINATION OF THE KINETIC PARAMETERS}

The kinetic rate coefficients of equations (eq. (15), (16), (17)) take the Arrhenius form of eq.(9) and require the determination of six more coefficients (i.e., $A_{j}$ and $E_{j}$, $\mathrm{j}=15,17$ where $\mathrm{j}$ is the reaction number. The formation of the specie $B_{1}$ (and therefore its subsequent branching) depends on the formation of species $Q_{1}$. A sensitivity analysis of the ignition delay predicted by the modified shell models confirms that the kinetic rate coefficients $k_{q 1}$ and $k_{b_{1}}$ of reactions (16) and (17) do not affect the numerical results significantly. Thus, literature values corresponding to low temperature oxidation reactions for 
a generic large hydrocarbon fuel have been used [20] for these parameters. As far as reaction (15) is concerned, the kinetic rate coefficient $k_{a}$ has been found to strongly affect ignition delay.

Theobald et al. [4] considered the kinetic path of reactions (4), (7) and (5) as the major contributor to the ignition delay in the diesel-like condition since the total delay is sensitive to the formation rate of intermediate specie $Q$ more than $R$ and $B$. Therefore, in the original shell model the parameter Af4, which governs the intermediate specie formation, is usually changed to fit diesel ignition data. Following a similar reasoning, the tuning of the modified shell model could be carried out by changing the rate of formation of both the intermediate species, $Q$ and $Q_{1}$, from which the branching agents $B$ and $B_{1}$ are generated. However, to the knowledge of the authors, there is no experimental evidence to prove the previous statement.

The prediction capability of the modified shell model has been checked with numerical simulations carried out for two different operating conditions: a homogeneously premixed constant volume combustion system and a small bore diesel engine configuration. Because of the fluid dynamics complexity of internal combustion engines, the comparison between the original and modified shell models has been first performed in the constant volume configuration (bomb-like case). For this set of simulations, the literature values suggested by Ranzi et al. [20] for the pre-exponential factor and the activation energy of reaction (15) have been used. For the engine simulations, the original shell model was tuned to match the experimental ignition delay only by changing the value of the $A_{f 4}$ pre-exponential factor. For the modified shell model, both $\mathrm{A}_{\mathrm{f} 4}$ and $k_{a}$ were adjusted to fit the experimental pressure traces.

\section{BOMB LIKE SIMULATIONS}

A single cell cubic mesh of side $5 \mathrm{~mm}$ has been used to simulate the ignition process under controlled conditions of temperature and pressure. A constant temperature wall was chosen as the boundary condition for the cell faces and the wall temperature was set equal to the initial temperature for all the operating conditions. The simulations were performed by comparing the ignition delay predicted by the original and modified shell models for different values of initial temperature, pressure and equivalence ratio. The effect of the pre-exponential factor of reaction (5) has also been investigated. These parameters have been changed one at a time over the ranges reported in Table 3 .

The results of the simulation are plotted in the 1000/T delay plane, where the data referred to the original and modified shell models are represented with solid and dotted lines, respectively. For all the investigated conditions, the ignition delay predicted by the modified shell is smaller than the delay predicted by the original shell model.

\begin{tabular}{|l|l|}
\hline Parameter & Range \\
\hline initial temperature & $650-1300 \quad[\mathrm{~K}]$ \\
\hline initial pressure & $2-6 \quad[\mathrm{MPa}]$ \\
\hline equivalence ratio & $0.50-1.50$ \\
\hline Af4 & $2.0 \times 103-2.0 \times 106$ \\
\hline
\end{tabular}

Table 3 - Ranges of variation for the bomb-like simulations

Since the original shell model has been found [21] to overestimate the ignition delay in the case of delayed injections, the results obtained in the present investigation can be considered quite interesting for future developments.

\section{EFFECT OF THE PRE-EXPONENTIAL FACTOR AF4}

The effect of the pre-exponential factor $A_{f 4}$ is illustrated in Figure 1 in the case of stoichiometric air-fuel ratio and for a pressure of $6 \mathrm{MPa}$.

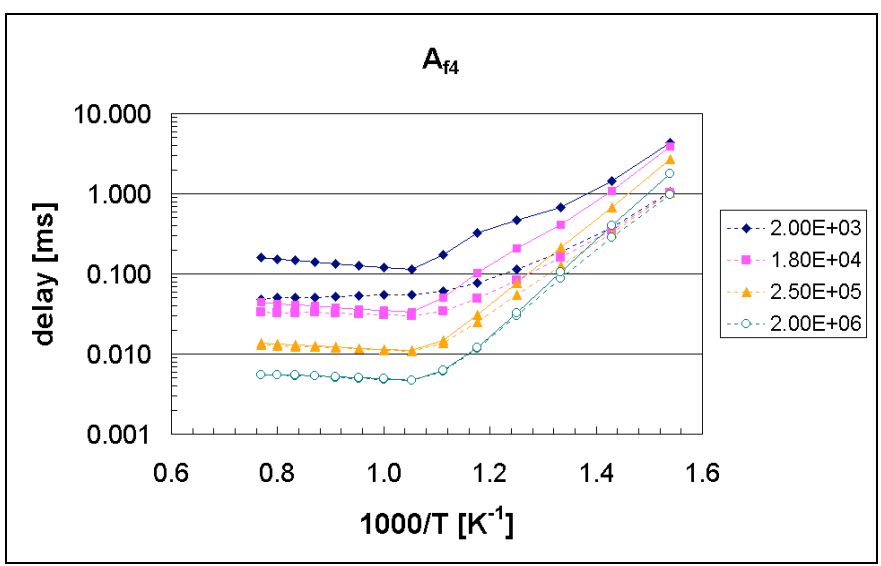

Figure 1 - Effect of the pre-exponential factor $A_{f 4}$ on the ignition delay

Data from both models show an increase of the ignition delay as temperature and $A_{f 4}$ diminishes. Note that for the highest values of $A_{f 4}$, the two models give the same values of ignition delay when temperature is higher than $800 \mathrm{~K}$. Such a behavior can be explained with the increase of the rate coefficient $f_{4}$ as temperature and $A_{f 4}$ are increased (see eq.(11)). For the highest values of $f_{4}$, the rate of formation of the intermediate specie $Q$ (eq. (5) ) becomes very high and the propagation steps due to this agent becomes predominant on the propagation path (eq. (15) and (16)) due to the intermediate specie $Q_{1}$ which is only present in the modified shell model. Therefore its effect on the ignition delay becomes negligible and the two models give the same results. For temperatures lower than $800 \mathrm{~K}$, the importance of propagation steps given by eq. (15) and (16)increases and the modified shell model predicts an earlier ignition because of the low temperature kinetics. As the temperature decreases below $700 \mathrm{~K}$, the propagation path due to $Q$ is quite negligible compared with the low temperature oxidation kinetics and the ignition delay predicted by the modified shell model is not affected by the pre-exponential factor Af4. 


\section{EFFECT OF THE INITIAL PRESSURE}

The effect of the initial pressure on the ignition delay for a value of $A_{f 4}=1.8 \times 10^{4}$ and a stoichiometric air-fuel ratio has been investigated. Results for the original and the modified shell models are reported in Figure 2 and Figure 3, respectively.

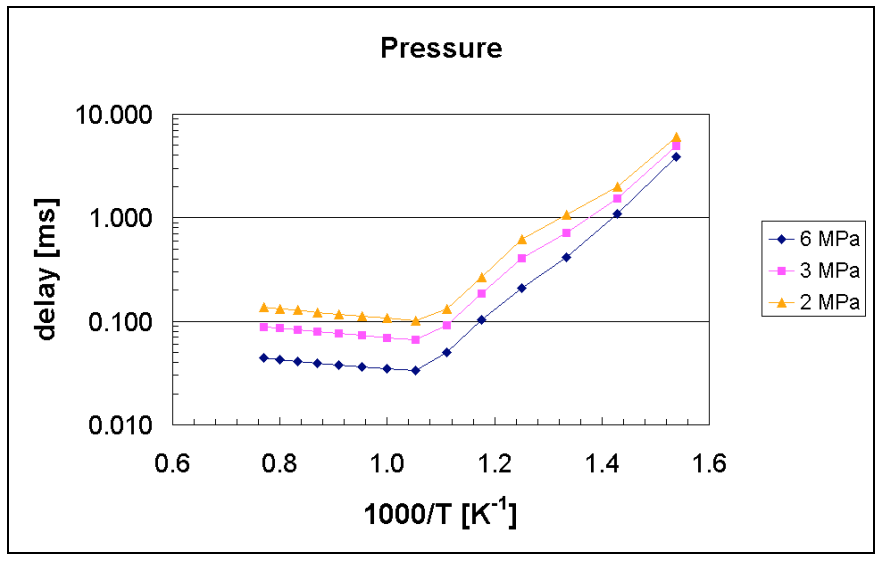

Figure 2 - Effect of the initial pressure on the ignition delay predicted by the original shell model

To explain the pressure effect on the ignition delay, it is necessary to recall the theory of thermal spontaneous ignition. According to this theory, ignition occurs when the rate of thermal energy release is greater than the rate of thermal energy dissipation [22]. The critical value of the rate of temperature growth in the cell (i.e., the value after which spontaneous ignition is observed) can be expressed as:

$$
\left(\frac{d T}{d t}\right)_{\text {citical }}=\frac{\dot{q}_{r}-\dot{q}_{w}}{c_{v} V} \geq 0
$$

where $\dot{q}_{r}$ is the amount of heat released due to chemical reaction per unit time, $\dot{q}_{w}=h S\left(T-T_{0}\right)$ represents the heat loss to the cell walls of area $S, c_{v}$ is the molar constant volume heat capacity and $\mathrm{V}$ is the cell volume. Note that the heat transfer coefficient $h$ is only weakly dependent on pressure and for sake of simplicity it is assumed to be constant for the following discussion. The heat release rate, $\dot{q}_{r}$, can be written in a simplified overall form as:

$\dot{q}_{r}=\dot{w} V Q=\rho^{n} \varepsilon^{n} A \exp (-E / R T) V Q$

where $\dot{w}$ is the reaction rate, $Q$ is the thermal energy release of the reaction, $\rho$ and $\varepsilon$ are the total density and the mass fraction of the reacting species, respectively, and $n$ is the overall reaction order. $A$ and $E$ are the preexponential and the activation energy in the simple rate constant expression. From the previous considerations, an exponential pressure dependence of the ignition delay on the pressure is expected. Compared with the results of the original shell model (Figure 2), the plots of the ignition delay of Figure 3 predicted by the modified shell model, are more consistent with the physics described above.

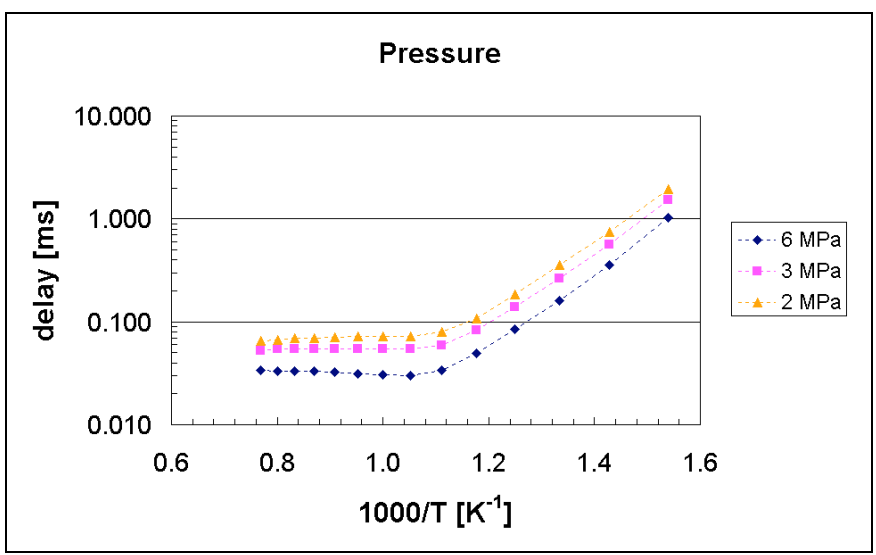

Figure 3 - Effect of the initial pressure on the ignition delay predicted by the modified shell model

\section{EFFECT OF EQUIVALENCE RATIO}

The effect of the equivalence ratio on ignition delay for a pressure of $3 \mathrm{MPa}$ and for a value of $A_{f 4}=1.8 \times 10^{4}$ is illustrated in Figure 4 . The graph shows that both models predict a reduction of the ignition delay as the air-fuel ratio, and therefore $\varepsilon$, increases. The results obtained by these simulations are consistent with the consideration carried out in the previous section.

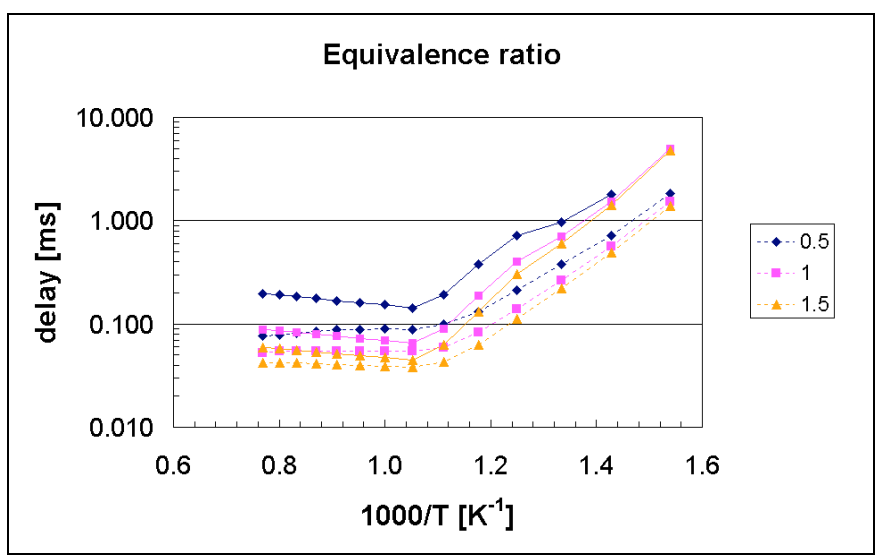

Figure 4 - Effect of the equivalence factor on the ignition delay

\section{ENGINE SIMULATIONS}

Three dimensional computations of a small bore commercial diesel engine have been performed and pressure traces and emissions levels produced by both the original and the modified shell model have been 
compared with the experimental data. Engine specifications are summarized in Table 4.

\begin{tabular}{|l|l|}
\hline Number of cylinders & 4 \\
\hline Bore & $88.0[\mathrm{~mm}]$ \\
\hline Stroke & $88.4[\mathrm{~mm}]$ \\
\hline Connecting rod length & $149[\mathrm{~mm}]$ \\
\hline Compression ratio & 19 \\
\hline Intake valves closing (IVC) & $173^{\circ}$ BTDC \\
\hline No nozzles & 6 \\
\hline Nozzle diameter & $169[\mu \mathrm{m}]$ \\
\hline Injection system & Common Rail \\
\hline
\end{tabular}

Table 4- Engine specifications.

To reduce the computational time, a 60 degree mesh consisting of 20 cells in the radial direction, 20 cells in the longitudinal direction and 37 cells in the axial direction, with a minimum of 17 cells at TDC, has been used. The cross section of the computational grid at TDC is reported in Figure 5. Experimental data for an engine speed of $2800 \mathrm{rpm}$ have been used to study the ignition delay predicted by both the modified and the original shell model as injection timing is varied from 15 degrees before TDC to $1^{\circ}$ TDC. The investigated operating conditions are reported in Table 5.

From the analysis performed in the bomb-like case, the ignition delay can be said to be significantly affected by pressure, temperature and equivalence ratio. It follows that ignition delay in diesel engines is strictly related to injection timing. CFD simulations of diesel engine carried out by using the shell model [21] exhibit the shortcoming of this model in predicting the ignition delay in the case of late injections. In this case, the low temperature kinetics plays a key role in the oxidation process, due to the temperature drop during the expansion stroke. Therefore, the ignition delay predicted by the original shell model becomes inaccurate for injections occurring after top dead center.

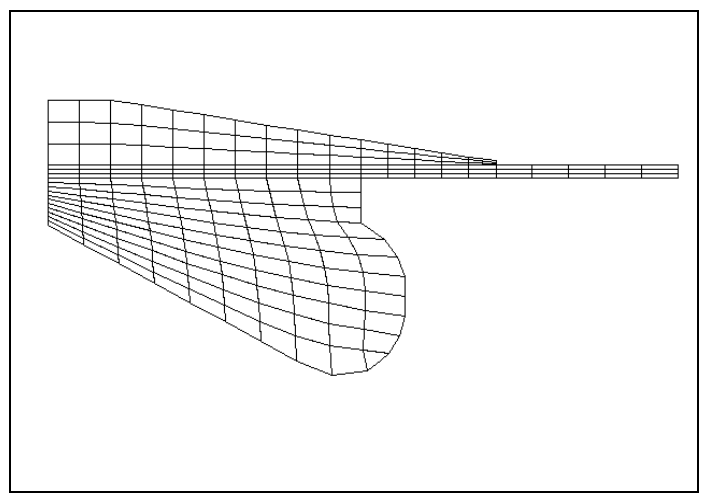

Figure 5 - The computational grid for engine simulations at TDC

The values of the kinetic constants were optimized to match the ignition delay of the experimental pressure traces. The original shell model has been found not to be able to predict ignition for case 5 when values of $A_{f 4}$ lower than $2.0 \times 10^{7}$ were set. Thus, this value has been chosen for the original shell model. The modified shell model performed best when the values $A_{f 4}=2.0 \times 10^{4}$, $A_{f a}=3.0 \times 10^{11}$ and $E_{f a}=3.0 \times 10^{4}$ were set. However, the reader must be aware that the above values were obtained by fitting engine pressure traces and they are not based on physical considerations. More detailed experimental data are necessary to find reference values for $E_{f a}$ and $A_{f a}$.

\begin{tabular}{|l|l|}
\hline Total injected mass & $27.8[\mathrm{mg}]$ \\
\hline Injection duration & $17.4^{\circ}$ \\
\hline Pressure at IVC & $0.17[\mathrm{MPa}]$ \\
\hline Temperature at IVC & $325[\mathrm{~K}]$ \\
\hline Engine speed & $2800[\mathrm{rpm}]$ \\
\hline Start of injection & Case $1=15$ BTDC \\
& Case $2=11^{\circ}$ BTDC \\
& Case $3=7^{\circ}$ BTDC \\
& Case $4=3^{\circ}$ BTDC \\
& Case $5=1^{\circ}$ ATDC \\
\hline
\end{tabular}

Table 5 - Engine operating conditions

The data of Figure 6 show the comparison between the numerical and experimental ignition delay versus injection timing. The ignition delay has been defined as the time between the start of injection and the onset of the heat release rate traces.

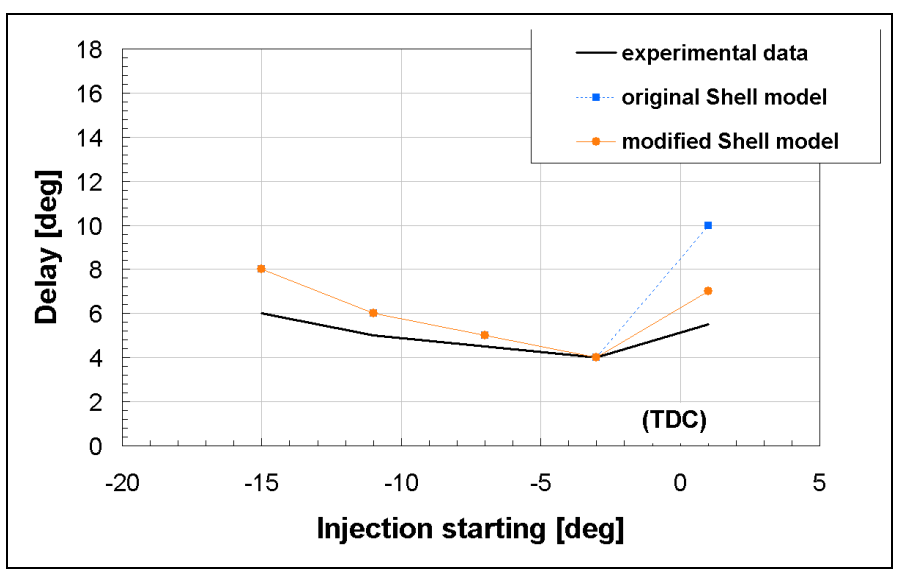

Figure 6 - Ignition delay versus injection starting

Note that for early injections the original and the modified shell models give very similar results. When fuel is injected after TDC, the modified shell model is still able to reasonably capture the ignition delay, whereas the original model overestimates it by about a factor 2 . Pressure traces for case 5 are shown in Figure 7. 


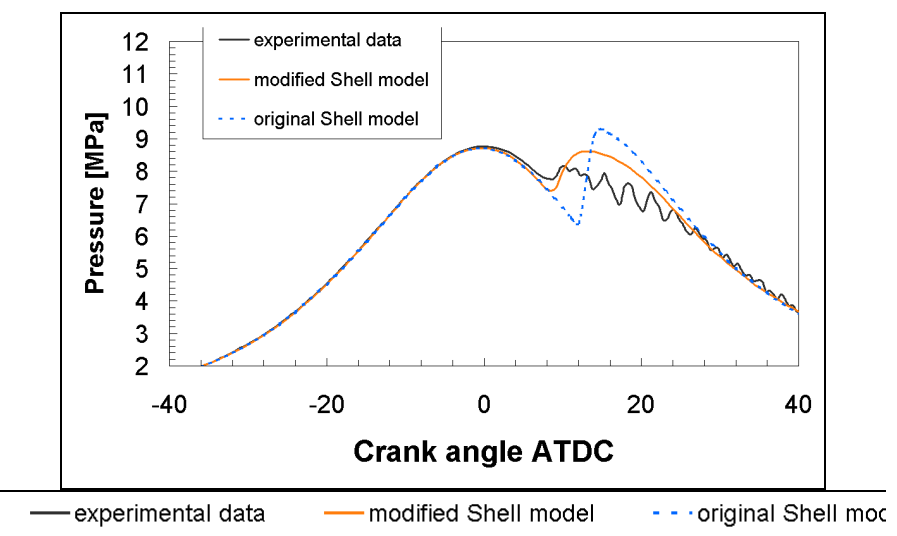

Figure 7 - Pressure traces for cases 1-5

As far as emissions are concerned, the NO-soot tradeoff obtained with the modified and original shell model have been compared and the results are shown in Figure 8. In the case of early injections, no major differences can be observed between the two models.

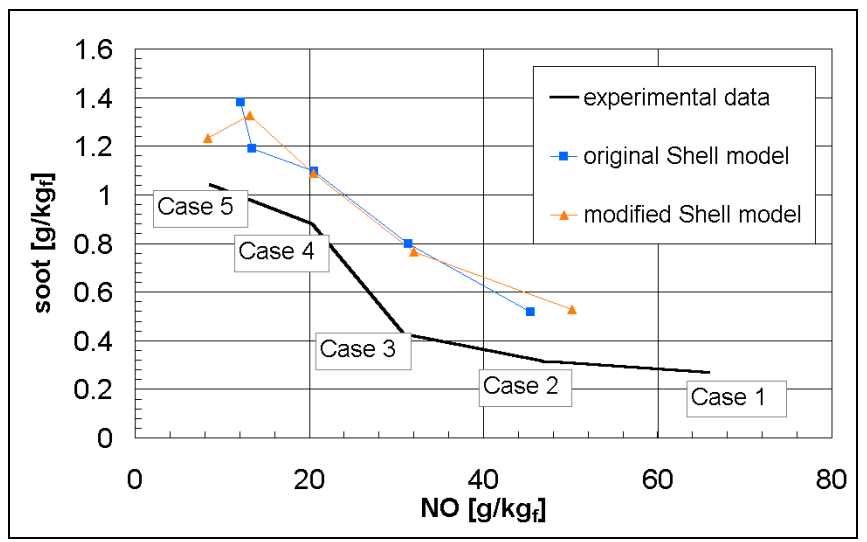

Figure 8 - Engine emissions tradeoffs

\section{CONCLUSION}

The results obtained with a modified version of the Shell model which takes into account the low temperature oxidation scheme of hydrocarbon fuels have been presented.

First, simulations were performed by comparing the ignition delay predicted by the original and modified shell models for different values of initial temperature, pressure and equivalence ratio in the case of a homogeneously premixed constant volume system. For all the investigated conditions, the modified shell model predicts a faster ignition process compared with the original shell model. Moreover, the modified shell model predicts more consistently the influence of pressure and equivalence ratio on the ignition delay. The influence of the rate coefficient $A_{f 4}$ on the results of both the original and the modified shell models has been investigated and it was found to be less effective in changing the ignition delays in the modified shell model.

Three-dimensional diesel engine simulations were also carried out to check the capability of the modified shell model to predict ignition delay versus injection timing trade-off. It has been found that the modified shell model gives interesting results in the case of delayed injection. The best performance was obtained by changing both $\mathrm{A}_{\mathrm{f} 4}$ and the kinetic rate coefficients of the reactions added to the original shell scheme. Further investigations are necessary to find reference values for these kinetic parameters.

The low temperature reactions have been found not to affect significantly the emissions tradeoffs obtained by the KIVA 3V code. However, simulations have shown significant changes in the emissions when different values of the kinetic rate coefficients were set.

\section{REFERENCES}

1. Halstead, M.P., Kirsch, L.J., Prothero, A., Quinn, C.P., "A Mathematical Model for Hydrocarbon Autoignition at High Pressures", Proc. R. Soc. Lond., A. 346, 515-538, 1975;

2. Halstead, M.P., Kirsch, A., Quinn, C.P., "The Autoignition of Hydrocarbon Fuels at High temperature and Pressure - Fitting of a Mathematical Model", Combustion and Flame, 30, 45-60, 1977;

3. Schäpertöns, H., Lee, W., "Multidimensional Modelling of Knocking Combustion in S.I. Engines", SAE paper 850502, 1985;

4. Theobald, M.A., Cheng, W. K., "A Numerical Study of Diesel Ignition", ASME Paper 87-FE-2, 1987;

5. Kong, S.C., Reitz, R.D., "Multidimensional modeling of Diesel Ignition and Combustion using a Multistep Kinetics Model", ASME Paper 93-ICE-22, 1993;

6. Cox, R.A., Cole, J.A., Combustion and Flame, 60, 109, 1985;

7. Hu, H., Keck, J., SAE Technical Paper 872110, 1987;

8. Gaballo, M.R., "Analisi e modellizzazione del fenomeno dell'autoaccensione in condizioni di bassa temperatura nei motori a combustione interna", Tesi di Laurea in Macchine, Università degli studi di Lecce, 2000 (in italian);

9. De Risi, A., Donateo, T., Laforgia, D., "Numerical Investigation of the Influence of physical parameters on Soot and NOx engine emissions", ASME ICE 2001, Spring Technical Conference, April 29 - May 2, Philadelphia, USA, 2001

10. Han, Z., Reitz, R. D. , 1995, "Turbulence Modeling of Internal Combustion Engines Using RNG-k-e Models", Combustion Science Technology, vol. 106, pp. 207; 
11. Amsden, 1989, KIVA 3 - A KIVA Program with Block-Sructured Mesh for Complex Geometries, Los Alamos National Labs,;

12. Dukowicz, J. K, 1980, "A Particle-Fluid Numerical Model for Liquid Sprays", Journal of Computational Physics, 35, pp. 229-253;

13. Beale, J. C., Reitz, R.D. , 1999, “ Modeling Spray Atomization with the Kelvin-Helmoltz/Rayleigh Taylor Hybrid Model", Atomization and sprays, Vol. 9, pp 623-650;

14. Abraham, J., Bracco, F.V., Reitz. R.D., "Comparison of Computed and Measured Premixed Charge Engine Combustion", Combust. Flame, 60, pp 309322, 1985;

15. Hiroyasu, H., Nishida, K., "Simplified Three Dimensional Modeling of Mixture Formation and Combustion in a D.I. Diesel Engine", SAE Paper 890269:

16. Nagle, J., Strickland-Constable, R. F. , 1962, "Oxidation of Carbon between 1000-2000 ${ }^{\circ} \mathrm{C}$ ", Procedure of the Fifth Carbon Conference, Vol. 1, Pergammon Press;

17. Hardenberg, H.O., Hase, F.W., "An Empirical Formula for Computing Pressure Rise Delay of a Fuel from its Cetane Number and from Relevant Parameters of D.I. Diesel Engines", SAE paper 790493, 1979;

18. Ayoub, N.S., Reitz, R.D., "Multidimensional Modeling of Fuel Composition Effects on Combustion and Cold-Starting in Diesel Engines", Sae Technical paper 952425, 1995;

19. Griffiths, J.F., "Reduced Kinetic Model and their Application to Practical Combustion Systems", Prog. Energy Combust. Sci., Vol. 21, pp. 25-107, 1995;

20. Ranzi, E., Faravelli, T., Gaffuri, P., Sogaro, A., "Low temperature combustion: Automatic Generation of Primary Oxydation Reactions and Lumping Procedures", Combustion and Flame, 102, 179-192, 1995;

21. A. De Risi, T. Donateo and D. Laforgia, "Theoretical Investigation on Variable Density Sprays", Internal Report, accepted to Atomization and sprays, 2001;

22. Irving Glassman, Combustion, Academic Press, San Diego, Ca, 1996. 